\title{
Editorial
}

\section{Good Antimicrobial Stewardship in the Hospital: Fitting, but Flagrantly Flagging}

\author{
Dale N. Gerding, MD
}

There are two general approaches to preventing or reversing antimicrobial resistance in the hospital setting. Prevention of transmission of resistant organisms from patient to patient is the traditional infection control approach that, if practiced perfectly, would resolve the problem of nosocomial infection due to resistant .organisms. It is the stuff of which all good hospital epidemiologists and infection control professionals are made. The second approach originates from the observation that the emergence of antimicrobial resistance is related to the use of antimicrobials. This approach is built on the premise that antimicrobial use (which is highly beneficial in the treatment of patients) is less than optimal and that, by improving (or reducing) use, there will occur a corresponding prevention or decline of antibiotic resistance. Both approaches were advocated in the "Guidelines for the Prevention of Antimicrobial Resistance in Hospitals" issued jointly in 1997 by the Society for Healthcare Epidemiology of America (SHEA) and the Infectious Diseases Society of America (IDSA). ${ }^{1}$

The guideline proposed four methods to influence antimicrobial use in order to prevent antimicrobial resistance. The first of these methods, optimal use of all antimicrobials, is the essence of what the guideline calls "good antimicrobial use stewardship": the practice of treating patients with the most effective, least toxic, and least costly antimicrobial for the precise duration of time needed to cure or prevent an infection. Some would call it the apple-pie-and-motherhood approach to antimicrobial prescribing. Clinical guidelines, treatment algorithms, and consultations provide the guidance for good stewardship. The second method recognizes the difficulty of accomplishing the ideal of good stewardship and advocates restriction, removal, or control of selected antimicrobials to reduce or prevent resistance. It is generally conceded to be the most effective method to change antimicrobial usage. ${ }^{1}$ The third proposed method, antimicrobial cycling, a preemptive form of cyclic restriction, was the subject of a January 2000 Supplement to this Journal, edited by Joseph John, Jr, MD, one of the authors of the SHEA/IDSA prevention guideline. ${ }^{2}$ The fourth suggested method, use of combinations of antimicrobials, is the basis for prevention of resistance development in the treatment of tuberculosis and human immunodeficiency virus infections, but has received little consideration for other hospital antimicrobial use.

In this issue of Infection Control and Hospital Epidemiology, Lawton and colleagues report the results of a survey of 47 Intensive Care Antimicrobial Resistance Epidemiology (ICARE) hospitals to determine the extent of their antimicrobial-use improvement practices. ${ }^{3}$ The questions were directed at methods 1 and 2 of the SHEA/IDSA guideline, querying the hospitals on practices (clinicalpractice guidelines) in place to support and improve good stewardship and on the presence of formulary controls and restrictions or limitations (stop orders) on antimicrobial use. Questions specifically regarding antimicrobial-cycling policies and use of combination therapy were not asked.

One of the most impressive aspects of the survey is the $100 \%$ completion rate. The only antibiotic practice found in all 47 hospitals was the presence of a pharmacy formulary, which in 4 of the hospitals was the only practice in place. Unfortunately, the presence of a formulary does not indicate the degree to which antimicrobial use is constrained in an institution. Restrictions on the use of any antimicrobial were in place in $40 \%$ of the hospitals. It is not clear that the formulary restrictions were in place to improve antibiotic use, since much of formulary restriction traditionally has had cost control as its major goal. Nonetheless, the restrictions were in place, and automatic stop orders were in place in $60 \%$ of hospitals. Finally, in the

From the Medical Service, Veterans' Affairs Chicago Healthcare System-Lakeside Division and Department of Medicine, Northwestern University Medical School, Chicago, Illinois.

Address reprint requests to Dale $N$. Gerding, $M D$, ican, INC, 12982 Valley View Rd, Eden Prairie, MN 55344.

OO-ED-020. Gerding DN. Good antimicrobial stewardship in the hospital: fitting, but flagrantly flagging. Infect Control Hosp Epidemiol 2000;21:253-255. 
area of improving stewardship, 70\% of hospitals had in place clinical-practice guidelines that addressed either diagnosis or use of antimicrobials.

Several observations can be made from these survey results: (1) antimicrobial-use restrictions, which together with formulary control are the most effective methods to alter antimicrobial use, ${ }^{1}$ were in place in only $40 \%$ of surveyed hospitals, suggesting that the most effective tool was used least; (2) rigorous use of restriction policies assured a high level of compliance with the "process change" of achieving use reduction, an essential step on the way to achieving the desired "outcome change" of resistance reduction.

Several studies published in the past decade suggest that removal of a specific antimicrobial from use in a specific environment is a highly successful strategy to reduce resistance to that antimicrobial and can eliminate both an outbreak and the clone of resistant bacteria involved. ${ }^{4-6}$ These outbreaks were caused by clindamycin-resistant Clostridium difficile and ended abruptly when clindamycin use was restricted effectively. ${ }^{5,6}$ Each was associated with a clone of clindamycin-resistant $C$ difficile that was distinct and that declined when clindamycin use was restricted. ${ }^{4-6}$ One could argue that this is an event that is uniquely tied to a specific organism-drug resistance pair that is not likely to be duplicated with other resistant organisms. I am inclined to disagree, because I am convinced by previous studies of the restriction of gentamicin that the removal of an antimicrobial from the environment can result in an abrupt and sustainable decrease in resistance. ${ }^{7}$ Success of such antimicrobial-restriction initiatives should in no way diminish the need to continue the practice of good infection control in the hospital environment. It is unlikely that any antimicrobial-restriction policy can succeed if widespread cross-infection due to poor infection control practices is present.

Nosocomial infections due to $C$ difficile and to vancomycin-resistant enterococci (VRE) share a similar epidemiology. The list of risk factors common to both infections includes antimicrobial exposure, number of antimicrobials, days of antimicrobial use, specific antimicrobials (third-generation cephalosporins, clindamycin, and imipenem) used, patient age, length of hospitalization, severity of underlying illness, use of electronic rectal thermometers and enteral feedings, environmental contamination, and contamination of the hands of healthcare workers. ${ }^{8}$ The specific risks that differ for the two infections are vancomycin exposure (the most frequently identified risk for VRE) and gastrointestinal colonization. ${ }^{8}$ Although both infections are highly associated with gastrointestinal colonization, colonization with $C$ difficile appears to be protective, whereas for VRE it is an added infection risk..$^{9,10}$ Why this digression? The purpose is to suggest that restriction of vancomycin may present the same opportunity to reduce VRE rates as restriction of clindamycin has for clindamycinresistant $C$ difficile. What has been missing is a suitable substitute for vancomycin, the only effective agent today for most methicillin-resistant Staphylococcus aureus and coagulase-negative staphylococcal infections. The rapid development and availability of new agents active against resistant gram-positive organisms, such as quinupristindalfopristin, linezolid, and daptomycin, may present the opportunity for vancomycin restriction in the near future. We need to be prepared to test vancomycin restriction (removal) for its effect on VRE rates when the opportunity becomes available. Let's get ready now.

The practice of good antimicrobial-use stewardship should be influenced by clinical-practice guidelines. Lawton et al found that such guidelines were present in $70 \%$ of the 47 ICARE hospitals. ${ }^{3}$ What was not determined in the survey was the extent to which these guidelines are successfully followed and, even more importantly, any process change or resistance outcome change that might follow from using clinical-practice guidelines. Clinical pathways often are employed to ensure guidelines are followed, but this is a labor-intensive practice, and improvement in quality has not been established by the use of clinical pathways. My bias is that good antimicrobial stewardship is not likely to be practiced consistently until a means is devised to deliver guideline-specific information to the practitioner at the time the prescribing decision is being made. Computerized patient information and decision support systems such as the one used at Latter Day Saints Hospital in Salt Lake City may provide this capability in the future. ${ }^{11}$ In the meantime, consistent practice of good stewardship in this area depends upon individual practitioner education, the ability of the practitioner to retain that information, and then using it to change practice patterns. Given the already overburdened schedules of physicians, we are probably naive to think that this can be accomplished for more than the most rudimentary of guidelines.

Thus, until technology enables us to provide credible cutting-edge decision support, I believe the most practical current way to influence antimicrobial use will be through antimicrobial restrictions of one kind or another. If American hospitals resemble ICARE hospitals, then $60 \%$ of them do not have any restrictions on antimicrobial use and as a result may have difficulty implementing restrictions. There may be good reasons for this. Teaching hospitals were found by Lawton et al to be significantly more likely than other hospitals to have restrictions in place (odds ratio, $12.0 ; 95 \%$ confidence interval, $1.9-75.9) .{ }^{3}$ In private practice, it is much more difficult to enforce restrictions, because either there is no one trained in infectious diseases available to approve restricted drugs, or the "antibiotic police" are frequently the same infectious disease specialists who must depend upon their colleagues to provide them with consultations. The approver of restricted antibiotics may be placed in a conflicted position of risking losing future consultations by denial of the restricted antimicrobial or may be suspected of soliciting additional consultations through the approval process. Furthermore, the role is often played without compensation, an added disincentive to undertake this responsibility.

Despite their shortcomings, I do not believe clinicalpractice guidelines are likely to diminish. Implementation 
of clinical-practice guidelines for antimicrobial use will remain a high priority; but, to be successfully implemented, the guidelines will need to be kept simple and focused, and the $30 \%$ of hospitals that do not use them will need to start. Nonetheless, I believe progress toward the goal of good antimicrobial stewardship will be slow until technology gives it the needed boost. At the rate that the Internet and hand-held wireless browser capability is developing, the breakthroughs may be sooner than we think. Meanwhile, good infection control practice remains as critically essential as it always will, but I'm also planning for the day when we can give vancomycin a rest with the use of new alternative agents. I can't wait to see if VRE rates drop as a result.

\section{REFERENCES}

1. Shlaes DM, Gerding DN, John JF Jr, Craig WA, Bornstein DL, Duncan $\mathrm{RA}$, et al. Society for Healthcare Epidemiology of America and Infectious Diseases Society of America Joint Committee on the Prevention of Antimicrobial Resistance: guidelines for the prevention of antimicrobial resistance in hospitals. Infect Control Hosp Epidemiol 1997;18:275-291.

2. Supplement on antibiotic cycling. Infect Control Hosp Epidemiol 2000;21(suppl):S1-S43.

3. Lawton RM, Fridkin SK, Gaynes RP, McGowan JE Jr. Practices to improve antimicrobial use at 47 US hospitals: the status of the 1997 SHEA/IDSA position paper recommendations. Infect Control Hosp Epidemiol 2000;21:256-259.

4. Johnson S, Samore MH, Farrow KA, Killgore GE, Tenover FC, Lyras D, et al. Epidemics of diarthea caused by a clindamycin-resistant strain of Clostridium difficile in four hospitals. N Engl J Med 1999;341:1645-1651.

5. Pear SM, Williamson TH, Bettin KM, Gerding DN, Galgiani, JN. Decrease in nosocomial Clostridium difficile-associated diarrhea by restricting clindamycin use. Ann Intern Med 1994;120:272-277.

6. Climo MW, Israel DS, Wong ES, Williams D, Coudron P, Markowitz SM. Hospital-wide restriction of clindamycin: effect on the incidence of Clostridium difficile-associated diarrhea and cost. Ann Intern Med 1998;128:989-995.

7. Gerding DN, Larson TA, Hughes RA, Weiler M, Shanholtzer C, Peterson LR. Aminoglycoside resistance and aminoglycoside usage: ten years of experience in one hospital. Antimicrob Agents Chemother 1991;351:1284-1290.

8. Gerding DN. Is there a relationship between vancomycin-resistant enterococcal infection and Clostridium difficile infection? Clin Infect Dis 1997;25(suppl 2):S206-S210.

9. Shim JK, Johnson S, Samore MH, Bliss DZ, Gerding DN. Primary symptomless colonization by Clostridium difficile and decreased risk of subsequent diarrhoea. Lancet 1998;351:633-636.

10. Edmond MB, Ober JF, Weinbaum DL, Pfaller MA, Hwang T, Sanford MD, et al. Vancomycin-resistant Enterococcus faecium bacteremia: risk factors for infection. Clin Infect Dis 1995;20:1126-1133.

11. Evans RS, Pestotnik SL, Classen DC, Clemmer TP, Weaver LK, Orme JF, et al. A computer-assisted management program for antibiotics and other antiinfective agents. $N$ Engl J Med 1998;338:232-238. 